Bull. Soc. math. France

130 (1), 2002, p. 101-121

\title{
STRUCTURE DU GROUPE DE GROTHENDIECK ÉQUIVARIANT D'UNE COURBE ET MODULES GALOISIENS
}

\author{
PAR NIELS BORNE
}

RÉSumÉ. - Cet article est consacré à l'étude de la structure d'anneau du groupe de Grothendieck équivariant d'une courbe projective munie d'une action d'un groupe fini. On explicite cette structure en introduisant un groupe de classes de cycles à coefficients dans les caractères et une notion d'auto-intersection pour ces cycles. De ce résultat, on déduit une expression de la caractéristique d'Euler équivariante d'un $G$-faisceau.

ABStRACT (Structure of the equivariant Grothendieck group of a curve and Galois modules)

This article is devoted to the study of the ring structure of the equivariant Grothendieck group of a projective curve provided with an action of a finite group. We make this structure explicit thanks to the introduction of a group of cycle classes with coefficients in the characters and a notion of self-intersection for theses cycles. From this result, we deduce an expression for the equivariant Euler characteristic of a $G$-sheaf.

\section{Table des matières}

1. Introduction ........................................... 102

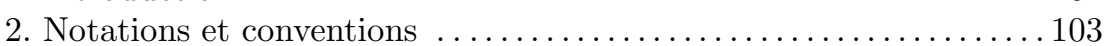

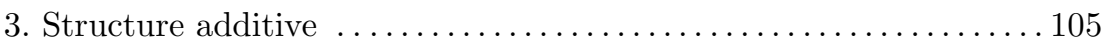

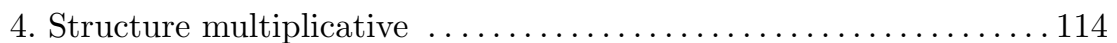

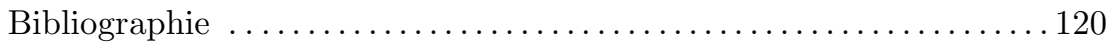

Texte reçu le 5 janvier 2001, accepté le $1^{\text {er }}$ juin 2001

Niels Borne, Laboratoire de Mathématiques Pures de Bordeaux, Université Bordeaux I, 351, cours de la Libération, F-33405 Talence Cedex (France) • E-mail : borne@math.ubordeaux.fr • Url : http://www.math.u-bordeaux.fr/ ${ }^{\text {borne }}$

Classification mathématique par sujets (2000). — 14H37, 14L30, 14C40.

Mots clefs. - Automorphismes des courbes, Actions de groupes sur des variétés ou des schémas, Théorèmes de Riemann-Roch. 


\section{Introduction}

L'objet de ce travail est l'étude des modules galoisiens sur les courbes. Plus précisément, si $X$ est une courbe projective et lisse sur un corps algébriquement clos $k$, munie d'une action d'un groupe fini $G$, et si $\mathcal{F}$ est un $G$-faisceau cohérent sur $X$, on se propose d'étudier $H^{0}(X, \mathcal{F})$ comme représentation de $G$.

Tel quel, ce problème est encore loin d'être élucidé. Par exemple, dans le cas où la théorie de la représentation est modulaire, il semble qu'on ne sache pas exprimer la classe d'isomorphisme de $H^{0}\left(X, \Omega_{X}\right)$ en fonction d'invariants de $X$ et de l'action (c'est-à-dire les invariants codant la ramification du morphisme quotient $\pi: X \rightarrow X / G)$.

Par contre, il est connu depuis le milieu des années 1980 que dans le cas d'une action dite modérée, on sait calculer la caractéristique d'Euler équivariante d'un $G$-faisceau cohérent $\chi(G, \mathcal{F})=\left[H^{0}(X, \mathcal{F})\right]-\left[H^{1}(X, \mathcal{F})\right]$ dans l'anneau des caractères $R_{k}(G)$ du groupe $G$ (voir [3], [10]). Toutefois, les formules explicites données par ces auteurs ne sont pas pleinement satisfaisantes : il n'est en effet pas clair en quoi elles sont un relèvement de la formule de Riemann-Roch usuelle par le morphisme de dimension $R_{k}(G) \rightarrow \mathbb{Z}$.

Pour remédier à ce problème, une approche naturelle est l'utilisation des propriétés fonctorielles de la $K$-théorie équivariante. Dans le cas d'une action triviale, la connaissance d'un isomorphisme explicite (donné par rang et déterminant) $K_{0}(X) \simeq \mathbb{Z} \oplus \operatorname{Pic} X$ et de l'isomorphisme réciproque permet de déduire la formule de Riemann-Roch usuelle. Dans le cas équivariant, le morphisme analogue n'est plus injectif. On surmonte cette difficulté en introduisant un groupe de cycles à coefficients équivariants, noté $A_{0}(G, X)$, se surjectant dans $\operatorname{Pic}_{G} X$. On définit de plus un morphisme naturel $\mathbb{Z} \oplus A_{0}(G, X) \rightarrow K_{0}(G, X)$ qui est en fait un isomorphime de groupes (voir le théorème 3.12 ). Ce résultat permet de définir une classe de Chern pour laquelle la formule de Riemann-Roch usuelle est valable dans le cadre équivariant.

En munissant $A_{0}(G, X)$ d'une structure d'anneau convenable, dont le produit est défini en termes d'auto-intersection de 0-cycles, on montre que l'isomorphisme ci-dessus peut se lire comme un isomorphisme d'anneaux unitaires (voir le théorème 4.10). De cette description, on déduit qu'un certain faisceau qu'on baptise ici faisceau canonique $\mathcal{C}_{X}:=\Omega_{X} \oplus \Omega_{X}^{\otimes 2} \oplus \cdots \oplus \Omega_{X}^{\otimes \# G}$ possède une caractéristique d'Euler équivariante particulièrement simple. En effet, lorsque l'action est modérée, on a :

$$
\chi\left(G, \mathcal{C}_{X}\right)=\# G \chi\left(\Omega_{X}\right)[k[G]]
$$

(voir théorème 4.13).

Je tiens à remercier Boas Erez qui, en relisant cet article, m'a aidé à l'améliorer.

TOME $130-2002-\mathrm{N}^{\mathrm{O}} 1$ 


\section{Notations et conventions}

Dans tout ce qui suit, $G$ désigne un groupe fini et $k$ un corps algébriquement clos.

2.1. $G$-courbe. - On appelle $G$-schéma tout schéma $X$ muni d'une action de $G$. Plus précisément un $G$-schéma est un couple $(X, \phi)$ où $\phi: G \rightarrow$ Aut $X$ est un morphisme de groupes. Lorsque, comme dans la plupart des cas, il n'y a pas d'ambiguïté, on omet de noter le morphisme $\phi$.

Une $G$-courbe $X$ sur $k$ est un $G$-schéma dont le schéma sous-jacent est une courbe algébrique projective et lisse sur $k$, au sens par exemple de [5], ch. IV. Par commodité, et bien que ça ne soit pas indispensable, on supposera que l'action est fidèle et fixe le corps $k$. La $G$-courbe $X$ admet un quotient dans la catégorie des schémas qu'on notera $\pi: X \rightarrow Y=X / G$. Le schéma $Y$ est en fait également une courbe projective et lisse sur $k$, le morphisme $\pi$ est fini, et l'extension des corps de fonctions associée est galoisienne de groupe de Galois $G$.

\section{2. $G$-faisceaux. — Soit $X$ un $G$-schéma.}

DÉfinition 2.1. - Soit $\mathcal{F}$ un faisceau (d'ensembles, de groupes,...) sur $X$. On appelle $G$-linéarisation de $\mathcal{F}$ la donnée d'une collection $\left(\psi_{g}\right)_{g \in G}$ de morphismes de faisceaux (d'ensembles, de groupes, ...) $\psi_{g}: g_{*} \mathcal{F} \rightarrow \mathcal{F}$ vérifiant les conditions suivantes :

1) $\psi_{1}=\mathrm{Id}$;

2) $\psi_{h g}=\psi_{h} \circ h_{*}\left(\psi_{g}\right)$ (condition de cocycle); autrement dit, le diagramme suivant commute :

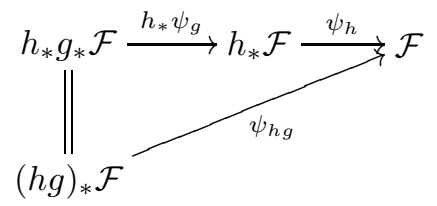

Un $G$-faisceau sur $X$ est un faisceau muni d'une $G$-linéarisation.

DÉfinition 2.2. - Pour tout $G$-schéma $X$, on notera $\operatorname{Coh}(G, X)$ la catégorie dont les objets sont les $G$-faisceaux cohérents sur $X$, et les morphismes les morphismes de $G$-faisceaux.

DÉFinition 2.3. - Le groupe de Picard équivariant de $X$, noté $\operatorname{Pic}_{G} X$, est le groupe des classes de $G$-isomorphismes de $G$-faisceaux inversibles sur $X$, muni du produit induit par le produit tensoriel. 


\subsection{Groupes de Grothendieck équivariants}

\subsubsection{Les groupes $G_{0}$ et $K_{0}$}

DÉfinition 2.4. - Soit $X$ un $G$-schéma noethérien. On désigne par $G_{0}(G, X)$ (resp. $\left.K_{0}(G, X)\right)$ le groupe abélien libre engendré par les classes d'isomorphismes $[\mathcal{F}]$ de $G$-faisceaux cohérents (resp. de $G$-faisceaux localement libres de rang fini) sur $X$, modulo les relations $[\mathcal{F}]=\left[\mathcal{F}^{\prime}\right]+\left[\mathcal{F}^{\prime \prime}\right]$ s'il existe une suite exacte de $G$-faisceaux

$$
0 \rightarrow \mathcal{F}^{\prime} \longrightarrow \mathcal{F} \longrightarrow \mathcal{F}^{\prime \prime} \rightarrow 0 \text {. }
$$

Par exemple le groupe $G_{0}(G$, Spec $k)$ est le groupe de Grothendieck des $k[G]$-modules de type fini. On le notera $R_{k}(G)$. Il est connu (voir [12]) que ce groupe s'identifie au groupe des caractères de Brauer de $G$.

Le groupe $K_{0}(G, X)$ est de plus muni naturellement d'une multiplication induite par le produit tensoriel des faisceaux, qui en fait un anneau commutatif unitaire.

Comme on le rappelle au moment de l'étude de cette structure, les deux groupes $G_{0}(G, X)$ et $K_{0}(G, X)$ coïncident lorsque le $G$-schéma considéré est séparé et régulier. Dans le cas des $G$-courbes étudiées ici, cela nous permettra de parler sans ambiguïté du groupe de Grothendieck équivariant de la courbe considérée.

On renvoie à [13] pour la définition des groupes de $K$-théorie équivariante supérieurs, et pour la démonstration de leur propriétés fondamentales. On les notera $G_{n}(G, X)$ (resp. $\left.K_{n}(G, X)\right)$.

2.3.2. Fonctorialité. - Le foncteur $G_{0}(G, \cdot)\left(\operatorname{resp} . K_{0}(G, \cdot)\right)$ est naturellement contravariant par rapport aux $G$-morphismes plats (resp. arbitraires) de $G$-schémas noethériens. De plus tout $G$-morphisme propre

$$
f: X \longrightarrow Y
$$

de $G$-schémas noethériens induit un morphisme de groupes

$$
f_{*}: G_{0}(G, X) \rightarrow G_{0}(G, Y)
$$

défini par la formule

$$
f_{*}([\mathcal{F}])=\sum_{n}(-1)^{n}\left[R^{n} f_{*}(\mathcal{F})\right] .
$$

En particulier, lorsque $X$ est une $G$-variété projective sur $k$, le morphisme structurel induit un morphisme $G_{0}(G, X) \rightarrow R_{k}(G)$ qu'on appelera caractéristique d'Euler équivariante, et qu'on notera $\chi(G, \cdot)$.

\subsubsection{Formule de projection}

Proposition 2.5. - Soit $f: X \rightarrow Y$ un $G$-morphisme propre de G-schémas noethériens, $x \in G_{0}(G, X), y \in K_{0}(G, Y)$. On a alors :

$$
f_{*}\left(x f^{*}(y)\right)=f_{*}(x) y \text {. }
$$

TOME $130-2002-\mathrm{N}^{\mathrm{O}} 1$ 
Démonstration. - La preuve classique (par exemple [1]) s'adapte au cas équivariant.

\section{Structure additive}

Pour tout le reste de ce travail, à l'exception du paragraphe 3.2 .2 , on supposera que $X$ est une $G$-courbe.

\subsection{Groupe de classes de cycles à coefficients équivariants}

\subsubsection{G-cycles}

DÉfinition 3.1. - On appelle $G$-cycle sur $X$ toute somme formelle de points fermés de $X$ du type

$$
D=\sum_{P \in X} V_{P} \cdot P
$$

vérifiant :

1) $V_{P}$ est un caractère de Brauer du groupe d'inertie $G_{P}$ de $P$ (c'est-à-dire $\left.V_{P} \in R_{k}\left(G_{P}\right)\right)$, nul sauf pour un nombre fini de points ;

2) si $P^{\prime}=g P$ pour $g \in G$, alors les caractères $V_{P}$ et $V_{P^{\prime}}$ sont conjugués (ce qu'on notera $V_{P^{\prime}}=V_{P}^{g}$ ).

On dira que $D$ est de plus effectif si tous les caractères $V_{P}$ sont des caractères de représentations des $G_{P}$. On notera $Z_{0}(G, X)$ le groupe abélien des $G$-cycles sur $X$.

REMARque 3.2. - D'une manière plus formelle, on a un isomorphisme canonique

$$
Z_{0}(G, X) \simeq \underset{S}{\lim _{S}} G_{0}(G, S)
$$

où $S$ varie parmi toutes les $G$-sous-variétés (fermées réduites) strictes de $X$.

DÉfinition 3.3. - On appellera $G$-degré et on notera

$$
\operatorname{deg}_{G}: Z_{0}(G, X) \longrightarrow R_{k}(G)
$$

le morphisme limite inductive des morphismes images directes $G_{0}(G, S) \rightarrow$ $R_{k}(G)$ induits par les morphismes structurels $S \rightarrow$ Spec $k, S$ variant parmi toutes les $G$-sous-variétés fermées strictes de $X$.

3.1.2. Classe de $G_{0}$-théorie associée à un $G$-cycle. — On définit un morphisme

$$
\gamma: Z_{0}(G, X) \longrightarrow G_{0}(G, X)
$$

de la manière suivante : si $D$ est un $G$-cycle à support dans $S, \gamma(D)$ est l'image de $D$ par le morphisme image directe $G_{0}(G, S) \rightarrow G_{0}(G, X)$ induit par l'immersion fermée $S \rightarrow X$. 
Lemme 3.4. - Soit $D$ un $G$-cycle sur $X$. On a l'égalité dans $R_{k}(G)$ :

$$
\chi(G, \gamma(D))=\operatorname{deg}_{G} D .
$$

Démonstration. - Soit $S$ le support de $D$ et $i: S \rightarrow X$ l'immersion fermée correspondante. On considère le diagramme commutatif

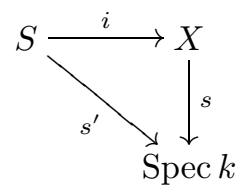

où $s$ et $s^{\prime}$ désignent les morphismes structurels. Il suffit de montrer que le diagramme induit au niveau des groupes $G_{0}$ commute, autrement dit que $s_{*} \circ i_{*}=s_{*}^{\prime}$. Mais ceci est une conséquence directe du fait que $i$ étant une immersion fermée, on a les égalités entre foncteurs $R^{n} s_{*}^{\prime}=R^{n} s_{*} \circ i_{*}$ pour $n \geq 0$ et $R^{n} i_{*}=0$ pour $n>0$.

\subsubsection{Image réciproque des cycles du quotient}

DÉFINITION 3.5. - On définit le morphisme image réciproque à valeurs dans les $G$-cycles associé au morphisme quotient $\pi: X \rightarrow Y=X / G$ comme étant le morphisme $\pi^{*}: Z_{0}(Y) \rightarrow Z_{0}(G, X)$ vérifiant pour tout point $Q$ de $Y$ :

$$
\pi^{*}(Q)=\sum_{P \rightarrow Q}\left[k\left[G_{P}\right]\right] \cdot P .
$$

Dans la suite de ce paragraphe, on va montrer que cette définition est naturelle du point de vue de la $K$-théorie. Plus précisément, si on restreint le morphisme ci-dessus aux cycles à support dans $T$ (une sous-variété stricte de $Y$ fixée), on obtient un morphisme

$$
\pi^{*}: G_{0}(T) \longrightarrow G_{0}(G, S)
$$

où $S=\left(\pi^{-1} T\right)_{\text {red }}$.

D'autre part, la courbe $Y$ étant lisse, le morphisme quotient

$$
\pi: X \longrightarrow Y=X / G
$$

est plat (voir [5], ch. III, prop. 9.7). De plus un faisceau tiré par ce morphisme est naturellement un $G$-faisceau. On dispose donc d'un foncteur exact entre catégories de $\left(G\right.$-)faisceaux cohérents $\pi^{*}: \operatorname{Coh}(Y) \rightarrow \operatorname{Coh}(G, X)$. Ce foncteur induit un morphisme $\pi^{*}: G_{0}(Y) \rightarrow G_{0}(G, X)$, qu'il convient de comparer à celui qu'on vient de définir.

Proposition 3.6. - Soit $j: T \rightarrow Y$ une sous-variété stricte de $Y$, $S=\left(\pi^{-1} T\right)_{\text {red }}, i: S \rightarrow X$ l'immersion fermée correspondante. Le diagramme

TOME $130-2002-\mathrm{N}^{\mathrm{O}} 1$ 
de groupes suivant est commutatif :

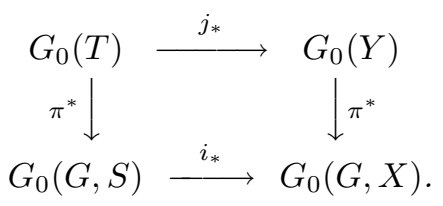

Démonstration. — Le diagramme commutatif

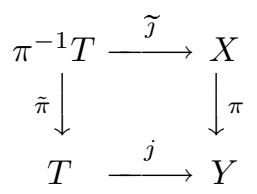

induit un diagramme commutatif de foncteurs exacts :

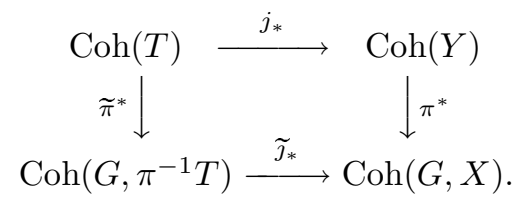

Ceci résulte du fait que $\pi$ est plat et que $j$ est une immersion fermée. En conséquence on a un diagramme commutatif de groupes :

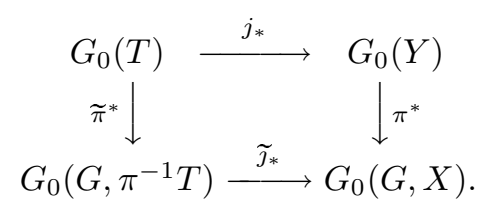

On va à présent identifier $G_{0}(G, S)$ et $G_{0}\left(G, \pi^{-1} T\right)$.

L'isomorphisme entre ces deux groupes résulte du théorème de dévissage (voir [11], th. 4). En effet le morphisme naturel $S \rightarrow \pi^{-1} T$ permet de voir $\operatorname{Coh}(G, S)$ comme une sous-catégorie de $\operatorname{Coh}\left(G, \pi^{-1} T\right)$. Pour montrer que tout objet de $\operatorname{Coh}\left(G, \pi^{-1} T\right)$ admet une filtration finie dont les quotients sont dans $\operatorname{Coh}(G, S)$, on peut supposer que $T$ est réduit à un point : $T=Q$. Soit alors $P$ un point de $X$ au-dessus de $Q, G_{P}$ son groupe d'inertie, $\mathcal{O}_{X, P}$ l'anneau local de $X$ en $P, \mathfrak{m}_{P}$ son idéal maximal, $e_{P}$ l'indice de ramification de $\pi$ en $P$. Alors la catégorie $\operatorname{Coh}\left(G, \pi^{-1} T\right)$ s'identifie à la catégorie $\operatorname{Coh}\left(G_{P}, \mathcal{O}_{X, P} / \mathfrak{m}_{P}{ }^{e_{P}}\right)$ et, de même, la catégorie $\operatorname{Coh}(G, S)$ s'identifie à la catégorie $\operatorname{Coh}\left(G_{P}, k\right)$. Alors, si $M$ est un $\mathcal{O}_{X, P} / \mathfrak{m}_{P}{ }^{e_{P}}$-module de type fini muni d'une action (tordue) de $G_{P}$, on dispose de la filtration naturelle $\left(\mathfrak{m}_{P}{ }^{n} M\right)_{n \geq 0}$, qui permet d'appliquer le théorème de dévissage, et de conclure à l'isomorphisme $G_{0}(G, S) \simeq G_{0}\left(G, \pi^{-1} T\right)$.

Pour conclure à la proposition, il suffit de noter deux choses. D'une part, via l'isomorphisme $G_{0}(G, S) \simeq G_{0}\left(G, \pi^{-1} T\right)$, le morphisme $\widetilde{\jmath}_{*}$ s'identifie au morphisme $i_{*}$. En effet, le morphisme composé $S \rightarrow \pi^{-1} T \rightarrow X$ n'est autre que $i$. D'autre part, toujours via l'isomorphisme $G_{0}(G, S) \simeq G_{0}\left(G, \pi^{-1} T\right)$, 
le morphisme $\widetilde{\pi}^{*}$ s'identifie au morphisme $\pi^{*}$. Pour cela, en se ramenant au cas où $T=Q$ comme ci-dessus, il suffit de voir que l'isomorphisme entre $G_{0}\left(G_{P}, \mathcal{O}_{X, P} / \mathfrak{m}_{P}{ }^{e_{P}}\right)$ et $G_{0}\left(G_{P}, k\right)=R_{k}\left(G_{P}\right)$ envoie $\mathcal{O}_{X, P} / \mathfrak{m}_{P}{ }^{e_{P}}$ sur $\left[k\left[G_{P}\right]\right]$. C'est évident dans le cas où la ramification est modérée. Le cas sauvage est un peu plus délicat, on renvoie à [2], prop. 4.8, pour les détails.

\subsection{4. Équivalence $Y$-rationnelle}

DÉfinition 3.7. - Les $G$-cycles principaux sur $X$ sont définis comme l'image réciproque, au sens du paragraphe précédent, des cycles principaux sur $Y$. Le groupe des classes de $G$-cycles pour l'équivalence $Y$-rationnelle sur $X$ est défini par

$$
A_{0}(G, X)=\frac{Z_{0}(G, X)}{\pi^{*}\left(\operatorname{Rat}_{k}(Y)\right)}
$$

où $\operatorname{Rat}_{k}(Y)$ est le groupe des cycles principaux sur $Y$.

Lemme 3.8. - Le diagramme suivant commute :

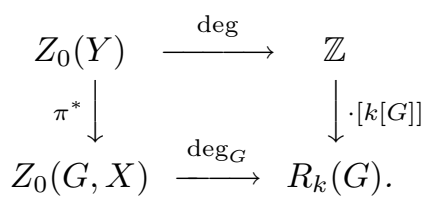

Démonstration. - Immédiat à partir des définitions.

DÉfinition 3.9. - On appelle $G$-degré d'une classe de $G$-cycles (pour l'équivalence $Y$-rationelle) le $G$-degré de n'importe quel élément de cette classe. On note (abusivement) $\operatorname{deg}_{G}: A_{0}(G, X) \rightarrow R_{k}(G)$ le morphisme correspondant.

On montre à présent que la notion de classe de $G_{0}$-théorie associée passe aussi au quotient. On note

$$
\gamma: Z_{0}(Y) \longrightarrow G_{0}(Y)
$$

le morphisme défini sur un diviseur effectif réduit $\delta$ par $\gamma(\delta)=\left[\mathcal{O}_{\delta}\right]$. Ce morphisme s'annulle sur les diviseurs principaux sur $Y$. En effet on a

$$
\gamma(\delta)=\left[\mathcal{O}_{Y}\right]-\left[\mathcal{L}_{Y}(-\delta)\right]
$$

pour tout diviseur $\delta$. Cette égalité équivaut au fait que son second membre est additif en $\delta$, ce qui résulte d'une part de [9], prop. 10.6, et d'autre part, $Y$ étant une courbe, $F_{\gamma}^{2} K_{0}(Y)=0$ (voir [9], th. 9.1).

Lemme 3.10. - Le diagramme suivant commute:

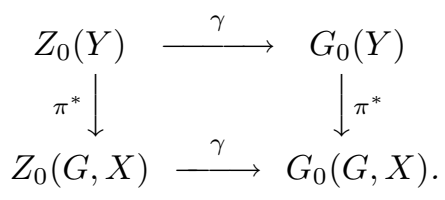

TOME $130-2002-\mathrm{N}^{\mathrm{O}} 1$ 
Démonstration. - C'est une conséquence directe de la proposition 3.6.

DÉfinition 3.11. - On note $\gamma: A_{0}(G, X) \rightarrow G_{0}(G, X)$ l'application déduite de $\gamma: Z_{0}(G, X) \rightarrow G_{0}(G, X)$ par passage au quotient.

3.1.5. Comparaison de $A_{0}(G, X)$ et $\operatorname{Pic}_{G} X$. - Ce paragraphe ne contient pas de preuve, mais le résultat énoncé ne sera pas réutilisé par la suite.

Pour comparer ces deux groupes, on commence par noter que $\operatorname{Pic}_{G} X$ s'interprète en termes de cycles (au sens classique) invariants sous l'action de $G$. Plus précisement on a un isomorphisme canonique (voir [2], th. 2.9) :

$$
\frac{Z_{0}(X)^{G}}{\pi^{*}\left(\operatorname{Rat}_{k} Y\right)} \simeq \operatorname{Pic}_{G} X
$$

Comme le morphisme surjectif $Z_{0}(G, X) \rightarrow Z_{0}(X)^{G}$ envoyant $\sum_{P} V_{P} \cdot P$ sur $\sum_{P} \operatorname{dim} V_{P} \cdot P$ passe au quotient on obtient un morphisme surjectif

$$
A_{0}(G, X) \longrightarrow \operatorname{Pic}_{G} X \text {. }
$$

Pour interpréter le noyau de ce morphisme, on peut montrer (en utilisant le théorème 3.12) qu'il s'identifie au morphisme naturel

$$
F_{\gamma}^{1} K_{0}(G, X) \longrightarrow \frac{F_{\gamma}^{1} K_{0}(G, X)}{F_{\gamma}^{2} K_{0}(G, X)} .
$$

Le groupe $F_{\gamma}^{2} K_{0}(G, X)$ est en général non trivial, il s'interprète par ailleurs uniquement en fonction des anneaux de caractères associés aux points à inertie non triviale de $X$.

\subsection{Structure additive du groupe de Grothendieck}

3.2.1. Énoncé

THÉORÈme 3.12. - On a un isomorphisme de groupes :

$$
\begin{aligned}
\phi: \mathbb{Z} \oplus A_{0}(G, X) & \longrightarrow G_{0}(G, X), \\
(r, D) & \longmapsto r\left[\mathcal{O}_{X}\right]+\gamma(D) .
\end{aligned}
$$

Ce résultat est bien connu dans le cas où l'action est triviale (voir par exemple [9], §10). La preuve du théorème est repoussée au paragraphe 3.2.3.

3.2.2. Foncteur $\pi_{*}^{G}$. — Dans ce paragraphe, les seules hypothèses faites sont que $X$ est un $G$-schéma noethérien admettant un quotient $\pi: X \rightarrow Y=X / G$ dans la catégorie des schémas.

DÉfinition 3.13. - Soit $\mathcal{F}$ un $G$-faisceau sur $X$. Le faisceau $\pi_{*}^{G}(\mathcal{F})$ est le faisceau sur $Y$ défini par

$$
\pi_{*}^{G}(\mathcal{F})(V)=\mathcal{F}\left(\pi^{-1} V\right)^{G}
$$

pour tout ouvert $V$ de $Y$.

BULLETIN DE LA SOCiÉtÉ MATHÉMATIQUe DE FRANCE 
Comme $\pi$ est fini, et $X$ est noethérien, le faisceau $\pi_{*}^{G}(\mathcal{F})$ est cohérent si $\mathcal{F}$ l'est. On obtient donc un foncteur $\pi_{*}^{G}: \operatorname{Coh}(G, X) \rightarrow \operatorname{Coh}(Y)$.

Le lemme ci-dessous est largement inspiré de [3].

LEMME 3.14. - (i) Le foncteur $\pi_{*}^{G}$ est adjoint à droite au foncteur $\pi^{*}$.

(ii) Pour tout $G$-faisceau cohérent $\mathcal{F}$ sur $X$ le morphisme naturel

$$
\pi^{*} \pi_{*}^{G} \mathcal{F} \longrightarrow \mathcal{F}
$$

est injectif de conoyau à support dans l'ensemble $\left\{P \in X \mid G_{P} \neq 1\right\}$.

(iii) Si l'action de $G$ sur $X$ est libre le foncteur $\pi^{*}: \operatorname{Coh}(Y) \rightarrow \operatorname{Coh}(G, X)$ est une équivalence de catégories.

Démonstration. - Le point (i) découle du fait que le foncteur $\pi_{*}^{G}$ se décompose :

$$
\operatorname{Coh}(G, X) \stackrel{\pi_{*}}{\longrightarrow} \operatorname{Coh}(G, Y) \stackrel{(\cdot)^{G}}{\longrightarrow} \operatorname{Coh}(Y)
$$

et du fait que $\pi^{*}$ se décompose en les foncteurs adjoints :

$$
\operatorname{Coh}(Y) \stackrel{\text { triv }}{\longrightarrow} \operatorname{Coh}(G, Y) \stackrel{\pi^{*}}{\longrightarrow} \operatorname{Coh}(G, X) .
$$

Ici, le foncteur triv est le foncteur qui associe à tout faisceau sur $Y$ le même faisceau muni de la $G$-linéarisation triviale.

Le point (ii) résulte du fait que si $P$ est un antécédent du point $Q$ par $\pi$, on a une identification naturelle de la complétion du germe du faisceau $\pi_{*}^{G} \mathcal{F}$ en $Q$ :

$$
\left(\widehat{\pi_{*}^{G \mathcal{F}}}\right)_{Q} \simeq \widehat{\mathcal{F}}_{P}^{G_{P}}
$$

Enfin, le point (iii) découle du point (ii) et du fait que pour tout faisceau cohérent $\mathcal{G}$ sur $Y$ le morphisme naturel $\mathcal{G} \rightarrow \pi_{*}^{G} \pi^{*} \mathcal{G}$ est dans ce cas un isomorphisme.

3.2.3. Démonstration du théorème 3.12. - On reprend les notations précisées au paragraphe 2 , en particulier la lettre $X$ désigne à nouveau une courbe. La démonstration la plus rapide consiste, suivant une idée de Lønsted [8], à faire intervenir la $K$-théorie supérieure :

LEMme 3.15. - Soit $j: T \rightarrow Y$ une sous-variété fermée stricte de $Y$ contenant le lieu de ramification du morphisme $\pi: X \rightarrow Y=X / G$. On note $V$ l'ouvert $Y-T, S=\pi^{-1} T_{\mathrm{red}}, i: S \rightarrow X$ la $G$-immersion fermée correspondante, $U$ l'ouvert $X-S$. On a un diagramme commutatif de groupes dont les lignes sont exactes :

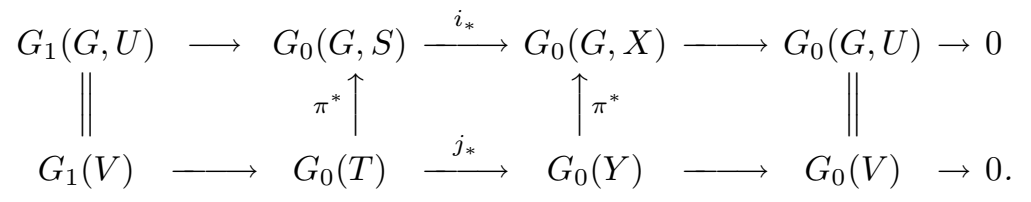

TOME $130-2002-\mathrm{N}^{\mathrm{O}} 1$ 
Démonstration. - Les lignes sont exactes car ce sont les suites de localisation pour les paires $(X, S)$ et $(Y, T)$. La commutativité du diagramme vient du fait que les suites de localisation sont naturelles pour les morphismes plats (voir [11], $\S 7.3$ pour le cas non-équivariant). Plus précisement, le morphisme $\pi$ induit un morphisme de la suite relative à la paire $(Y, T)$ vers la suite relative à la paire $\left(X, \pi^{-1} T\right)$, qui par dévissage s'identifie à la suite relative à la paire $(X, S)$. Enfin, les isomorphismes verticaux découlent du lemme 3.14, (iii).

On peut conclure à présent la démonstration du théorème 3.12. C'est essentiellement une chasse dans le diagramme ci-dessus.

Pour l'injectivité de $\phi$, soit un couple $(r, D)$ tel que $r\left[\mathcal{O}_{X}\right]+\gamma(D)=0$. En appliquant le morphisme rk : $G_{0}(G, X) \rightarrow \mathbb{Z}$, il vient $r=0$. Comme alors $\gamma(D)=0$ on a, d'après le lemme 3.15 appliqué à une sous-variété $T$ convenable, $D=\pi^{*}(\delta)$ pour un $\delta \in G_{0}(T)$ tel que $j_{*}(\delta)=0$. Or comme le résultat à montrer est vrai dans le cas où $G=1$ (autrement dit $\mathbb{Z} \oplus A_{0}(Y) \simeq G_{0}(Y)$ ), cette dernière égalité signifie que $\delta=0$ dans $A_{0}(Y)$ et montre donc que $D=0$ dans $A_{0}(G, X)$.

Pour la surjectivité de $\phi$, on fixe $x \in G_{0}(G, X)$, qu'on peut supposer de la forme $x=[\mathcal{F}]$ pour un $G$-faisceau cohérent $\mathcal{F}$ sur $X$. D'après le lemme 3.14 (ii), on a une suite exacte :

$$
0 \rightarrow \pi^{*} \mathcal{G} \longrightarrow \mathcal{F} \longrightarrow \mathcal{R} \rightarrow 0
$$

où $\mathcal{G}=\pi_{*}^{G} \mathcal{F}$ et $\mathcal{R}$ est un faisceau de torsion à support dans le lieu de ramification $X_{\text {ram }}$ du morphisme $\pi$. L'exactitude en $G_{0}(G, X)$ de la suite exacte de localisation pour la paire $\left(X, X_{\text {ram }}\right)$ montre que $[\mathcal{R}]$ est de la forme $\gamma(D)$ pour un certain $G$-cycle $D$, et donc dans l'image de $\phi$. D'autre part, le fait que le diagramme naturel

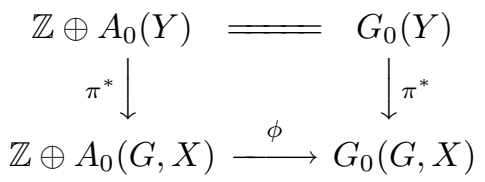

commute permet de conclure que $\left[\pi^{*} \mathcal{G}\right]$, et donc finalement $x$, est bien dans l'image de $\phi$.

3.3. Première classe de Chern équivariante. - Dans le cas où l'action est triviale, on connaît explicitement l'isomorphisme réciproque de l'isomorphisme du théorème 3.12 : celui-ci envoie $[\mathcal{F}]$ sur le couple $\left(\operatorname{rk} \mathcal{F}, c_{1}(\mathcal{F})\right)$. Ceci fournit une définition alternative de la première classe de Chern qui s'étend au cas équivariant :

DÉFINITION 3.16. — On note

$$
c_{1}: G_{0}(G, X) \longrightarrow A_{0}(G, X)
$$

BULletin DE LA SOCIÉtÉ MATHÉMATIQUE DE FRANCE 
et on appelle première classe de Chern équivariante, le morphisme composé par l'isomorphisme réciproque $G_{0}(G, X) \rightarrow \mathbb{Z} \oplus A_{0}(G, X)$ de l'isomorphisme du théorème 3.12 , suivi de la deuxième projection $\mathbb{Z} \oplus A_{0}(G, X) \rightarrow A_{0}(G, X)$.

LEMME 3.17. - Le diagramme suivant commute :

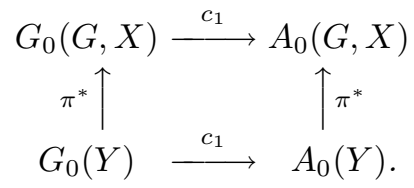

Démonstration. - C'est une conséquence du lemme 3.10 et de la définition de $c_{1}$.

\subsection{Formule de Riemann-Roch équivariante}

ThÉorème 3.18. - Soit $\mathcal{F}$ un $G$-faisceau cohérent sur $X$. On note $\chi(G, \mathcal{F})$ sa caractéristique d'Euler équivariante et $\operatorname{deg}_{G}(\mathcal{F})=\operatorname{deg}_{G}\left(c_{1}[\mathcal{F}]\right)$. On a alors l'égalité dans $R_{k}(G)$ :

$$
\chi(G, \mathcal{F})=\operatorname{rk}(\mathcal{F}) \chi\left(G, \mathcal{O}_{X}\right)+\operatorname{deg}_{G}(\mathcal{F}) .
$$

Démonstration. — D'après le théorème 3.12 on a l'égalité suivante dans $G_{0}(G, X)$ :

$$
[\mathcal{F}]=\operatorname{rk}(\mathcal{F})\left[\mathcal{O}_{X}\right]+\gamma\left(c_{1}[\mathcal{F}]\right)
$$

Le théorème découle alors du lemme 3.4.

\subsection{Première classe de Chern équivariante du faisceau des diffé- rentielles}

Proposition 3.19. - On suppose que l'action de $G$ sur la courbe $X$ est modérée (i.e. la caractéristique du corps de base $k$ ne divise pas l'ordre des groupes d'inertie). Alors on a l'égalité dans le groupe $A_{0}(G, X)$ :

$$
c_{1}\left[\Omega_{X}\right]=\pi^{*}\left(c_{1}\left[\Omega_{Y}\right]\right)+\sum_{P}\left(\left[k\left[G_{P}\right]\right]-[k]\right) \cdot P
$$

où $[k]$ désigne la classe de la représentation triviale de $G_{P}$.

Démonstration. - En tenant compte du lemme 3.17 on est ramené à calculer $c_{1}\left(\left[\Omega_{X}\right]-\left[\pi^{*} \Omega_{Y}\right]\right)$. Pour cela, on va exhiber une filtration décroissante de $\Omega_{X}$ à $\pi^{*} \Omega_{Y}$. Le morphisme injectif canonique $\pi^{*} \Omega_{Y} \rightarrow \Omega_{X}$ définit un faisceau d'idéaux $\pi^{*} \Omega_{Y} \otimes_{\mathcal{O}_{X}} \Omega_{X}^{-1}$, et le diviseur de Cartier correspondant est noté $R$. Autrement dit $R$ est le diviseur de ramification de l'action, i.e. le diviseur de ramification du morphisme $\pi: X \rightarrow Y=X / G$. Comme l'action est modérée, on peut expliciter $R$ seulement en fonction des indices de ramification de $\pi$,

TOME $130-2002-\mathrm{N}^{\mathrm{O}} 1$ 
de la manière suivante. Soient $O_{1}, \ldots, O_{n}$ les orbites ramifiées de $X$, considérées comme diviseurs réduits sur $X$, et $e_{1}, \ldots, e_{n}$ les indices de ramification correspondants. Alors

$$
R=\sum_{m=1}^{n}\left(e_{m}-1\right) O_{m}
$$

Cette égalité permet de voir que la suite de faisceaux

$$
\left(\Omega_{X} \otimes_{\mathcal{O}_{X}} \mathcal{L}_{X}\left(-\sum_{\ell=1}^{m}\left(e_{\ell}-1\right) O_{\ell}\right)\right)_{0 \leq m \leq n}
$$

est une filtration descendante de $\Omega_{X}$ à $\pi^{*} \Omega_{Y}$. En écrivant $\left[\Omega_{X}\right]-\left[\pi^{*} \Omega_{Y}\right]$ comme la somme des différences des classes dans $G_{0}(G, X)$ des termes consécutifs de cette suite, on voit que la proposition découle du lemme suivant.

LEMME 3.20. - Soit $O$ une orbite, d'indice de ramification e, considérée comme un diviseur réduit sur $X$, et $D$ un diviseur sur $X$ dont le support ne rencontre pas $O$. Si l'action de $G$ sur $X$ est modérée on a l'égalité dans $G_{0}(G, X)$ :

$$
\left[\Omega_{X}\right]\left(\left[\mathcal{L}_{X}(D)\right]-\left[\mathcal{L}_{X}(D-(e-1) O)\right]\right)=\gamma\left(\sum_{P \in O}\left(\left[k\left[G_{P}\right]\right]-[k]\right) \cdot P\right) .
$$

Démonstration. - On introduit une nouvelle filtration en écrivant :

$$
\left[\mathcal{L}_{X}(D)\right]-\left[\mathcal{L}_{X}(D-(e-1) O)\right]=\sum_{n=0}^{e-2}\left[\mathcal{L}_{X}(D-n O)\right]-\left[\mathcal{L}_{X}(D-(n+1) O)\right]
$$

Soit $i: O \rightarrow X$ l'immersion fermée de $O$ dans $X$. En tensorisant la suite exacte $0 \rightarrow \mathcal{L}_{X}(-O) \rightarrow \mathcal{O}_{X} \rightarrow i_{*}\left(\mathcal{O}_{D}\right) \rightarrow 0$ par $\mathcal{L}_{X}(D-n O)$, on voit que

$$
\left[\mathcal{L}_{X}(D-n O)\right]-\left[\mathcal{L}_{X}(D-(n+1) O)\right]=i_{*}\left(\left[\mathcal{N}_{O / X}^{\otimes n}\right]\right)
$$

où $\mathcal{N}_{O / X}$ désigne le faisceau conormal de $O$ dans $X$. Comme $O$ est de dimension 0 , on a $\mathcal{N}_{O / X} \simeq i^{*} \Omega_{X}$, et donc d'après la formule de projection :

$$
\left[\Omega_{X}\right] i_{*}\left(\left[\mathcal{N}_{O / X}^{\otimes n}\right]\right)=i_{*}\left(\left[\mathcal{N}_{O / X}^{\otimes n+1}\right]\right) .
$$

Pour expliciter ce terme, on note $\psi_{P}$ l'action de $G_{P}$ sur l'espace cotangent $\mathfrak{m}_{P} / \mathfrak{m}_{P}{ }^{2}$ à $X$ en $P, \psi_{P}$ est un générateur de $\widehat{G}_{P}=\operatorname{Hom}\left(G_{P}, k^{*}\right)$. D'autre part comme $\left(\mathcal{N}_{O / X}\right)_{P}=\mathcal{N}_{P / X} \simeq \mathfrak{m}_{P} / \mathfrak{m}_{P}{ }^{2}$ on a par définition de $\gamma$ et $\psi_{P}$ :

$$
i_{*}\left(\left[\mathcal{N}_{O / X}^{\otimes n+1}\right]\right)=\gamma\left(\sum_{P \in O} \psi_{P}^{n+1} \cdot P\right) .
$$

Le lemme s'en déduit en sommant sur $n$.

BULletin DE LA SOCIÉtÉ MATHÉMATIQUE DE FRANCE 


\section{Structure multiplicative}

Le premier paragraphe de cette section consiste en des rappels de résultats connus qui permettent d'utiliser ceux de la partie 3 .

\subsection{Comparaison de $K_{0}$ et $G_{0}$}

4.1.1. Comparaison des groupes. — Soit $S$ un $G$-schéma noethérien.

LEMME 4.1. - Le produit tensoriel induit une structure d'anneau unitaire sur $K_{0}(G, S)$.

Démonstration. — Ceci résulte simplement du fait qu'un faisceau localement libre est plat.

THÉORÈME 4.2. — Si S est un G-schéma noethérien régulier et séparé le morphisme naturel $K_{0}(G, S) \rightarrow G_{0}(G, S)$ est un isomorphisme.

Démonstration. - Voir [13], th. 5.7 ou [6], Satz 2.1.

4.1.2. Comparaison des comportements fonctoriels. - Le foncteur $K_{0}(G, \cdot)$ est contravariant par rapport aux $G$-morphismes arbitraires de $G$-schémas noethériens. D'autre part, on a vu au paragraphe 2 que le foncteur $G_{0}(G, \cdot)$ est naturellement covariant par rapport aux $G$-morphismes propres. Il est intéressant, dans ce cas, de comparer ces deux comportements fonctoriels.

On peut par exemple étudier le cas d'une immersion fermée $i: S \rightarrow S^{\prime}$ entre deux $G$-schémas noethériens réguliers et séparés. On a alors un diagramme :

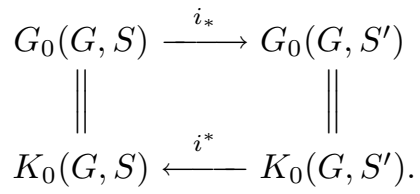

On est conduit à énoncer la formule d'auto-intersection :

ThÉORÈme 4.3. - Soit $i: S \rightarrow S^{\prime}$ une G-immersion fermée entre deux $G$ schémas noethériens réguliers et séparés. Soit de plus $\mathcal{N}_{S / S^{\prime}}$ le faisceau conormal associé et $\lambda_{-1}\left(\mathcal{N}_{S / S^{\prime}}\right)$ l'élément de $K_{0}(G, S)$ :

$$
\lambda_{-1}\left(\mathcal{N}_{S / S^{\prime}}\right)=\sum_{n \geq 0}(-1)^{n} \wedge^{n}\left[\mathcal{N}_{S / S^{\prime}}\right]
$$

Alors :

(i) pour tout $x$ dans $K_{0}(G, S): i^{*} i_{*} x=\lambda_{-1}\left(\mathcal{N}_{S / S^{\prime}}\right) x$;

(ii) pour tout $x, y$ dans $K_{0}(G, S): i_{*}\left(\lambda_{-1}\left(\mathcal{N}_{S / S^{\prime}}\right) x y\right)=i_{*} x i_{*} y$.

TOME $130-2002-\mathrm{N}^{\mathrm{O}} 1$ 
Remarque 4.4. - Pour un résultat dans un cadre plus général, et la définition des opérations lambda en $K$-théorie équivariante, voir [7], §3. Pour les besoins de ce travail, le théorème 4.3 sera amplement suffisant. Pour la commodité du lecteur, on rappelle le principe d'une preuve classique ci-dessous.

Démonstration. - On commence par remarquer que (ii) est une conséquence directe de (i) et de la formule de projection. Quant à (i), c'est une conséquence des deux faits suivants.

Premièrement, si $\mathcal{G}$ est un $G$-faisceau cohérent sur $S^{\prime}$, les faisceaux $\operatorname{Tor}_{n}^{\mathcal{O}_{S^{\prime}}}\left(i_{*} \mathcal{O}_{S}, \mathcal{G}\right)$ sont des faisceaux sur $S^{\prime}$, à support dans $S$. Si on les identifie avec des faisceaux sur $S$, on a la formule dans $K_{0}(G, S)$ :

$$
i^{*}[\mathcal{G}]=\sum_{n \geq 0}(-1)^{n}\left[\operatorname{Tor}_{n}^{\mathcal{O}_{S^{\prime}}}\left(i_{*} \mathcal{O}_{S}, \mathcal{G}\right)\right] .
$$

Cette formule résulte directement de la définition des foncteurs Tor. En effet, si $\mathcal{E} . \rightarrow \mathcal{G}$ est une résolution de $\mathcal{G}$ par des faisceaux localements libres, alors

$$
i^{*}[\mathcal{G}]=i^{*}\left(\sum_{n \geq 0}(-1)^{n}\left[\mathcal{E}_{n}\right]\right)=\sum_{n \geq 0}(-1)^{n}\left[i^{*}\left(\mathcal{E}_{n}\right)\right] .
$$

Cette quantité est donc la caractéristique d'Euler du complexe $i^{*}(\mathcal{E} \bullet) \rightarrow i^{*} \mathcal{G}$, qui est égale à la somme alternée de son homologie, d'où la formule donnée.

Deuxièmement, si $\mathcal{F}$ est $G$-faisceau localement libre sur $S$, alors on a un isomorphisme canonique :

$$
\operatorname{Tor}_{n}^{\mathcal{O}_{S^{\prime}}}\left(i_{*} \mathcal{O}_{S}, i_{*} \mathcal{F}\right) \simeq \mathcal{F} \otimes_{\mathcal{O}_{S}} \wedge^{n} \mathcal{N}_{S / S^{\prime}}
$$

Pour la construction de cet isomorphisme, voir par exemple [9], §3.

4.2. Produit de 0-cycles. - Si on veut que les $G$-cycles introduits pour expliquer la structure additive de $G_{0}(G, X)$ soient compatibles avec la structure multiplicative induite par $K_{0}(G, X)$, on doit considérer que ces $G$-cycles ont une auto-intersection non triviale, ce qui les distingue du cas classique où, les codimensions étant additives, un produit de 0-cycles est nul. Pour formaliser cette notion, on introduit dans ce paragraphe une structure d'anneau (non unitaire) sur $A_{0}(G, X)$. On peut encore noter que ce type de structure de intervient en $K$-théorie classique des schémas pour décrire la $K$-théorie des éclatements. Les notations employées ici sont en particulier inspirées de [4].

4.2.1. Anneau $K_{N}$. - Soit $K$ un $\lambda$-anneau au sens de [4], I, $\S 1$, et $N$ un élément positif. Alors l'expression

$$
\lambda_{-1}(N)=\sum_{n=0}^{\infty}(-1)^{n} \lambda^{n}(N)
$$

BULLETIN DE LA SOCIÉtÉ MATHÉMATIQUE DE FRANCE 
a un sens dans $K$, et on peut définir une nouvelle structure d'anneau sur $K$ en conservant l'addition telle quelle et en posant pour nouvelle multiplication :

$$
x_{N} y=\lambda_{-1}(N) x y .
$$

On notera $K_{N}$ cet anneau.

4.2.2. Produit sur $A_{0}(G, X)$. - Soit $i: S \rightarrow X$ une $G$-sous-variété stricte de $X$. On note $N_{S}=\left[\mathcal{N}_{S / X}\right]$ la classe du fibré conormal dans $K_{0}(G, S)$. Cette classe définit l'anneau $K_{0}(G, S)_{N_{S}}$.

Comme on a un isomorphisme de groupes abéliens $K_{0}(G, S) \simeq G_{0}(G, S)$ on peut transférer cette structure d'anneau à $G_{0}(G, S)$, et l'anneau obtenu est noté $G_{0}(G, S)_{N_{S}}$.

Proposition 4.5. - Soient $S$ et $S^{\prime}$ deux G-sous-variétés strictes de $X$ et $j: S \rightarrow S^{\prime}$ une $G$-immersion fermée. Le morphisme

$$
j_{*}: G_{0}(G, S)_{N_{S}} \longrightarrow G_{0}\left(G, S^{\prime}\right)_{N_{S^{\prime}}}
$$

est un morphisme d'anneaux.

Démonstration. - Il est clair que $\mathcal{N}_{S / S^{\prime}}=0$, et donc $\mathcal{N}_{S / X} \simeq j^{*} \mathcal{N}_{S^{\prime} / X}$, ce qui entraîne $\lambda_{-1}\left(N_{S}\right)=j^{*}\left(\lambda_{-1}\left(N_{S}^{\prime}\right)\right)$. En conséquence, si $x, y$ sont deux éléments de $G_{0}(G, S)$, alors d'après la formule de projection :

$$
j_{*}\left(x_{N_{S}}^{\dot{*}} y\right)=j_{*}\left(\lambda_{-1}\left(N_{S}\right) x y\right)=\lambda_{-1}\left(N_{S}^{\prime}\right) j_{*}(x y) .
$$

Comme $\mathcal{N}_{S / S^{\prime}}=0$, le théorème 4.3 (ii) donne $j_{*}(x y)=j_{*}(x) j_{*}(y)$, et donc

$$
\lambda_{-1}\left(N_{S}^{\prime}\right) j_{*}(x y)=\lambda_{-1}\left(N_{S}^{\prime}\right) j_{*}(x) j_{*}(y)=j_{*}(x) \underset{N_{S}^{\prime}}{\dot{ }} j_{*}(y),
$$

ce qui démontre le lemme.

Comme $Z_{0}(G, X) \simeq \lim _{S} G_{0}(G, S)$, la proposition ci-dessus justifie la définition :

DÉfinition 4.6. - On note $Z_{0}(G, X)_{N}$ le groupe $Z_{0}(G, X)$ muni de la structure d'anneau induite par celles des $G_{0}(G, S)_{N_{S}}$.

Proposition 4.7. - Dans $Z_{0}(G, X)_{N}$, le produit d'un élément de $\pi^{*}\left(Z_{0}(Y)\right)$ avec un élément quelconque est nul.

Démonstration. - Il suffit de prouver que si $Q$ est un point de $Y$, alors

$$
\pi^{*}(Q)=\sum_{P \rightarrow Q}\left[k\left[G_{P}\right]\right] P
$$

possède cette propriété. Soit $S=\left(\pi^{-1} Q\right)_{\text {red. }}$ Il suffit de montrer que si $D$ est un $G$-cycle à support dans $S$, alors $\pi^{*}(Q) \cdot N_{S} D=0$. C'est une conséquence facile du calcul explicite de $\lambda_{-1}\left(N_{S}\right)$ dans ce cas. En effet, soit $P$ un point de $S$ et $\psi_{P}$ l'action de $G_{P}$ sur l'espace cotangent en $X$ à $P$. Alors, si on identifie

TOME $130-2002-\mathrm{N}^{\mathrm{O}} 1$ 
$G_{0}(G, S)$ à $R_{k}\left(G_{P}\right), \lambda_{-1}\left(N_{S}\right)$ s'identifie à $1-\psi_{P}$ et l'égalité $\pi^{*}(Q) \cdot{ }_{N_{S}} D=0$ découle de l'égalité $\left(1-\psi_{P}\right)\left[k\left[G_{P}\right]\right]=0$.

DÉfinition 4.8. - On note $A_{0}(G, X)_{N}$ le groupe $A_{0}(G, X)$ muni de la structure d'anneau induite par celle de $Z_{0}(G, X)_{N}$.

\subsection{Structure multiplicative du groupe de Grothendieck}

4.3.1. Anneau unitaire associé à un anneau. - On rappelle le fait suivant :

Proposition 4.9. - Soit $A$ un anneau. Il existe un anneau unitaire $A^{+}$, unique à isomorphisme près, et un morphisme $A \rightarrow A^{+}$, tel que tout morphisme $A \rightarrow B$ de $A$ vers un anneau unitaire $B$ se factorise de manière unique par le morphisme $A \rightarrow A^{+}$.

Démonstration. - L'unicité découle du fait que $A^{+}$est défini par une propriété universelle. Pour l'existence, on considère l'anneau unitaire dont le groupe abélien est $\mathbb{Z} \oplus A$, muni du produit donné par :

$$
(r, a) \cdot\left(r^{\prime}, a^{\prime}\right)=\left(r r^{\prime}, r a^{\prime}+r^{\prime} a+a a^{\prime}\right)
$$

et le morphisme $A \rightarrow A^{+}$donné par $a \mapsto(0, a)$.

\subsection{2. Énoncé}

THÉORÈME 4.10. - Il existe un isomorphisme canonique d'anneaux unitaires

$$
A_{0}(G, X)_{N}^{+} \simeq K_{0}(G, X)
$$

uniquement déterminé par le fait que pour tout $G$-cycle $D$, l'image de $D$ est $\gamma(D)$.

4.3.3. Preuve. - Le morphisme $\gamma: A_{0}(G, X) \rightarrow G_{0}(G, X)$ défini à la section 3.11 et l'isomorphisme canonique de résolution $G_{0}(G, X) \simeq K_{0}(G, X)$ définissent un morphisme $A_{0}(G, X) \rightarrow K_{0}(G, X)$ qu'on note encore $\gamma$. Le théorème 4.10 découle du théorème 3.12 et du lemme suivant :

Lemme 4.11. - Le morphisme $\gamma: A_{0}(G, X)_{N} \rightarrow K_{0}(G, X)$ est un morphisme d'anneaux.

Démonstration. - Cela résulte de la définition du produit sur $A_{0}(G, X)_{N}$ et du théorème 4.3 , (ii).

\subsection{Caractéristique d'Euler équivariante du faisceau canonique}

4.4.1. Énoncé

DÉfinition 4.12. - On appelle faisceau canonique de la $G$-courbe $X$ le faisceau :

$$
\mathcal{C}_{X}:=\bigoplus_{l=1}^{\# G} \Omega_{X}^{\otimes \ell}=\Omega_{X} \oplus \Omega_{X}^{\otimes 2} \oplus \cdots \oplus \Omega_{X}^{\otimes \# G}
$$

BULletin DE LA SOCiÉtÉ MATHÉMATiQUe DE FRANCE 
ThÉORÈme 4.13. - Si l'action de $G$ sur $X$ est modérée on a dans $R_{k}(G)$ :

$$
\chi\left(G, \mathcal{C}_{X}\right)=\# G \chi\left(\Omega_{X}\right)[k[G]] .
$$

La démonstration de cette égalité est l'objet des paragraphes suivants.

4.4.2. Réduction du problème. - D'après le théorème de Riemann-Roch usuel sur les courbes, les deux représentations virtuelles figurant dans l'égalité à démontrer ont même dimension. En conséquence, il suffira de montrer que $\chi\left(G, \mathcal{C}_{X}\right) \equiv 0 \bmod [k[G]]$, autrement dit que $\chi\left(G, \mathcal{C}_{X}\right)$ est un multiple entier de $[k[G]]$ (il n'y a pas de différence entre le sous-groupe et l'idéal engendré par $[k[G]]$ dans $\left.R_{k}(G)\right)$. On peut donc raisonner modulo les éléments de $K_{0}(G, X)$ dont la caractéristique d'Euler équivariante est multiple de $[k[G]]$. Pour cette raison, on introduit le sous-groupe de $K_{0}(G, X)$ suivant :

$$
I=\left\{\pi^{*}(\gamma(\delta)) \mid \delta \in A_{0}(Y)\right\} .
$$

Comme d'après le lemme 3.4 on a $\chi\left(G, \pi^{*}(\gamma(\delta))\right)=\operatorname{deg} \delta[k[G]]$, le morphisme $\chi(G, \cdot)$ induit un morphisme de groupes $K_{0}(G, X) / I \rightarrow R_{k}(G) /[k[G]]$ qu'on note de la même manière. Tout revient donc à montrer que l'image de $\left[\mathcal{C}_{X}\right]$ par ce morphisme vaut 0 . On va démontrer le résultat un peu plus fort : si $\mathcal{O}_{X}[G]$ est l'image réciproque de $k[G]$ par le morphisme structurel $X \rightarrow$ Spec $k$, alors

$$
\left[\mathcal{C}_{X}\right] \equiv\left[\mathcal{O}_{X}[G]\right] \bmod I
$$

Comme d'après la formule de projection, $\chi\left(G, \mathcal{O}_{X}[G]\right)=\chi\left(\mathcal{O}_{X}\right)[k[G]]$, le théorème découlera de l'égalité (1). La suite de la démonstration utilise la structure multiplicative de $K_{0}(G, X)$, et en particulier le fait que $I$ est un idéal de cet anneau. En effet, si $x \in K_{0}(G, X)$ et $\delta \in A_{0}(Y)$, il résulte de la formule de projection que $x \pi^{*}(\gamma(\delta))=\operatorname{rk}(x) \pi^{*}(\gamma(\delta))$.

4.4.3. Première étape. - Dans un premier temps, on va montrer l'égalité suivante :

$$
\left(\left[\mathcal{O}_{X}\right]-\left[\Omega_{X}\right]\right)\left[\mathcal{C}_{X}\right] \equiv 0 \bmod I .
$$

En développant, le premier terme, on voit que cette égalité équivaut à $\left[\Omega_{X}^{\otimes \# G}\right] \equiv\left[\mathcal{O}_{X}\right] \bmod I$. Cette égalité résulte des deux faits suivants : premièrement, le morphisme naturel $\operatorname{Pic}_{G} X \rightarrow K_{0}(G, X)^{*}$ induit un morphisme

$$
\frac{\operatorname{Pic}_{G} X}{\pi^{*} \operatorname{Pic} Y} \longrightarrow\left(\frac{K_{0}(G, X)}{I}\right)^{*} ;
$$

deuxièmement, l'exposant du groupe $\operatorname{Pic}_{G} X / \pi^{*} \operatorname{Pic} Y$ divise $\# G$. Pour montrer le premier fait, soit $\mathcal{M}$ est un faisceau inversible sur $Y$, alors

$$
\begin{aligned}
{\left[\pi^{*} \mathcal{M}\right] } & =\pi^{*}[\mathcal{M}]=\pi^{*}\left(\left[\mathcal{O}_{Y}\right]+\gamma\left(c_{1}(\mathcal{M})\right)\right) \\
& =\left[\mathcal{O}_{X}\right]+\pi^{*}\left(\gamma\left(c_{1}(\mathcal{M})\right)\right) \equiv\left[\mathcal{O}_{X}\right] \bmod I .
\end{aligned}
$$

TOME $130-2002-\mathrm{N}^{\mathrm{O}} 1$ 
Le deuxième fait découle de l'existence d'un isomorphisme canonique (voir [2], th. 2.9) :

$$
\frac{Z_{0}(X)^{G}}{\pi^{*}\left(Z_{0}(Y)\right)} \simeq \frac{\operatorname{Pic}_{G} X}{\pi^{*} \operatorname{Pic} Y}
$$

4.4.4. Deuxième étape. - On va à présent montrer l'égalité suivante :

$$
\left(\left[\mathcal{O}_{X}\right]-\left[\Omega_{X}\right]\right)\left(\left[\mathcal{C}_{X}\right]-\left[\mathcal{O}_{X}[G]\right]\right) \equiv 0 \bmod I .
$$

Au vu de (2), cela équivaut à $\left(\left[\mathcal{O}_{X}\right]-\left[\Omega_{X}\right]\right)\left[\mathcal{O}_{X}[G]\right] \equiv 0 \bmod I$. Mais d'après le théorème $3.12,\left[\mathcal{O}_{X}\right]-\left[\Omega_{X}\right]=\gamma(D)$ pour un certain $G$-cycle $D$. De plus, si on note $\pi_{*}: A_{0}(G, X) \rightarrow A_{0}(Y)$ le morphisme qui envoie $\sum_{P} V_{P} \cdot P$ sur $\sum_{P} \operatorname{dim} V_{P} \cdot \pi(P)$, il découle de la formule de projection que

$$
\left[\mathcal{O}_{X}[G]\right] \gamma(D)=\gamma\left(\pi^{*}\left(\pi_{*} D\right)\right),
$$

ce qui achève de démontrer (3).

\subsubsection{Troisième étape}

Lemme 4.14. - Si pour un $x$ de $K_{0}(G, X)$ de rang nul, on a

$$
\left(\left[\mathcal{O}_{X}\right]-\left[\Omega_{X}\right]\right) x=0 \quad \text { dans } \quad K_{0}(G, X) / I,
$$

alors $x=0$ dans $K_{0}(G, X) / I$.

Démonstration. - On note $X_{\mathrm{ram}}=\left\{P \in X \mid G_{P} \neq 1\right\}$ le lieu de ramification de l'action et

$$
D_{\text {ram }}=\sum_{P \in X_{\text {ram }}} P
$$

le $G$-cycle réduit correspondant. La proposition 3.19 montre que

$$
c_{1}\left(\left[\Omega_{X}\right]\right) \equiv-D_{\text {ram }} \bmod \pi^{*}\left(A_{0}(Y)\right)
$$

et donc d'après le lemme $3.10, \gamma\left(c_{1}\left(\left[\Omega_{X}\right]\right)\right) \equiv-\gamma\left(D_{\text {ram }}\right) \bmod I$. Comme de plus d'après le théorème 3.12 on a $\left[\Omega_{X}\right]=\left[\mathcal{O}_{X}\right]+\gamma\left(c_{1}\left(\left[\Omega_{X}\right]\right)\right)$, il vient

$$
\left[\mathcal{O}_{X}\right]-\left[\Omega_{X}\right] \equiv \gamma\left(D_{\text {ram }}\right) \bmod I .
$$

Comme $x$ est de rang nul, on peut, d'après le théorème 3.12 , écrire $x=\gamma(D)$ pour un certain $G$-cycle $D$. L'hypothèse du théorème se traduit alors par

$$
\gamma\left(D_{\mathrm{ram}}\right) \gamma(D)=\pi^{*}(\gamma(\delta))
$$

pour un certain cycle $\delta$ sur $Y$. D'après le théorème 4.10 et le lemme 3.10 , cette égalité s'écrit encore $\gamma\left(D_{\mathrm{ram}}{ }_{N} D\right)=\gamma\left(\pi^{*} \delta\right)$, ou encore, d'après le théorème $3.12, D_{\mathrm{ram}}{ }^{N} D=\pi^{*} \delta$ dans $A_{0}(G, X)$. Quitte à changer $\delta$ par un diviseur $Y$-linéairement équivalent, on peut même supposer que cette égalité est valable dans $Z_{0}(G, X)$.

On écrit à présent

$$
D=\sum_{P \in X} \cdot V_{P} P \quad \text { et } \quad \delta=\sum_{Q \in Y} n_{Q} \cdot Q .
$$

BULletin DE LA SOCiÉtÉ MATHÉMATIQUE DE FRANCE 
Soit de plus $\psi_{P}$ le caractère par lequel $G_{P}$ agit sur l'espace cotangent à $X$ en $P$. En appliquant la définition du produit dans $Z_{0}(G, X)$ et en égalisant les coefficients des $G$-cycles ci-dessus on obtient

$$
\left(1-\psi_{P}\right) V_{P}=n_{\pi(P)}\left[k\left[G_{P}\right]\right]
$$

pour tout point $P$ de $X_{\text {ram}}$. Or en prenant les dimensions, on voit que ceci impose $n_{\pi(P)}=0$, et par suite $V_{P}$ est un multiple de $\left[k\left[G_{P}\right]\right]$. Le lemme est donc démontré.

Le lemme et l'égalité (3) montrent que l'égalité (1) est vraie, ce qui achève la démonstration du théorème 4.13 .

REMARQUe 4.15. - Des formules 3.18 et 4.13 on déduit l'expression

$$
\# G \chi\left(G, \mathcal{O}_{X}\right)=\# G \chi\left(\Omega_{X}\right)[k[G]]-\operatorname{deg}_{G} \mathcal{C}_{X}
$$

qui ramène le problème du calcul explicite de $\chi\left(G, \mathcal{O}_{X}\right)$ à celui du calcul de $c_{1}\left(\mathcal{C}_{X}\right)$. Ce calcul est possible, malgré la définition un peu artificielle de la première classe de Chern adoptée; on ne le présentera pas ici.

\section{BIBLIOGRAPHIE}

[1] Borel (A.) \& Serre (J.-P.) - Le théorème de Riemann-Roch, Bull. Soc. Math. France, t. 86 (1958), pp. 97-136.

[2] Borne (N.) - Une formule de Riemann-Roch équivariante pour les courbes, Preprint, 1999.

[3] Ellingsrud (G.) \& Lønsted (K.) - An equivariant Lefschetz formula for finite reductive groups, Math. Ann., t. 251 (1980), pp. 253-261.

[4] Fulton (W.) \& LAng (S.) - Riemann-Roch algebra, Grund. Math. Wissen., vol. 277, Springer-Verlag, 1985.

[5] Hartshorne (R.) - Algebraic geometry, Graduate Texts in Mathematics, vol. 52, Springer-Verlag, New York-Heidelberg, 1977.

[6] KöcK (B.) - Das Adams-Riemann-Roch-Theorem in der höheren äquivarianten $K$-Theorie, J. reine angew. Math., t. 421 (1991), pp. 189-217.

[7] _ The Grothendieck-Riemann-Roch theorem for group schemes actions, Ann. Sci. École Norm. Sup., t. 4-31 (1998), pp. 415-458.

[8] Lønsted (K.) - On $G$-line bundles and $K_{G}(X)$, J. Math. Kyoto Univ., t. 4-23 (1983), pp. 775-793.

[9] Manin (Ju. I.) - Lectures on the $K$-functor in algebraic geometry, russian : Uspehi Mat. Nauk, t. 5-24 (1969), pp.3-86, english : Russ. Math. Surv., t. 5-24 (1969), pp. 1-89.

TOME $130-2002-\mathrm{N}^{\mathrm{O}} 1$ 
[10] NAKAJIMA (S.) - Galois module structure of cohomology groups for tamely ramified coverings of algebraic varieties, J. Number Theory, t. 22 (1986), pp. 115-123.

[11] Quillen (D.) - Higher algebraic K-theory. I, in Algebraic K-theory, (Proc. Conf., Battelle Memorial Inst., Seattle, Washington, 1972), Lecture Notes in Math., vol. 341, Springer, Berlin, 1973, pp. 85-147.

[12] SERRE (J.-P.) - Représentations linéaires des groupes finis, Hermann, Paris, 1971.

[13] Thomason (R.W.) - Algebraic K-theory of group scheme actions, in Algebraic topology and algebraic K-theory (Princeton, N.J., 1983), Ann. Math. Stud., vol. 113, University Press, Princeton, NJ, 1987, pp. 539-563. 